\title{
Entre laïcité et neutralité : quelles frontières en droit du travail ?
}

\section{Isabelle Desbarats}

\section{(2) OpenEdition}

1 Journals

Édition électronique

URL : https://journals.openedition.org/rdr/684

DOI : $10.4000 /$ rdr.684

ISSN : 2534-7462

\section{Éditeur}

Presses universitaires de Strasbourg

\section{Édition imprimée}

Date de publication : 31 octobre 2017

Pagination : 51-67

ISBN : 978-2-86820-974-0

ISSN : 2493-8637

\section{Référence électronique}

Isabelle Desbarats, «Entre laïcité et neutralité : quelles frontières en droit du travail ? », Revue du droit des religions [En ligne], 4 | 2017, mis en ligne le 15 janvier 2020, consulté le 23 mai 2022. URL : http:// journals.openedition.org/rdr/684 ; DOI : https://doi.org/10.4000/rdr.684

\section{(c) (7) \&}

La revue du droit des religions est mise à disposition selon les termes de la Creative Commons Attribution - Pas d'Utilisation Commerciale 4.0 International - CC BY-NC 4.0. 


\section{ENTRE LÄ̈CITÉ ET NEUTRALITÉ : QUELLES FRONTIERES EN DROIT DU TRAVAIL ?}

\section{Isabelle DESBARATS}

Université Toulouse-1 Capitole, Centre de droit des affaires (CDA)

\section{RÉSUMÉ}

Alors que le fait religieux constitue une question de plus en plus sensible dans un contexte de recul de la pratique cultuelle mais de réaffirmation des identités religieuses, comment concilier exigences professionnelles et liberté religieuse dans les organisations privées? Quelle confrontation possible dans un environnement public marqué par le principe de laïcité, mais dans une société également plurielle, où la gestion de la diversité constitue un enjeu social ? Le principe de laïcité « à la française » peut-il être mobilisé pour résoudre les problèmes contemporains auxquels sont confrontées les entreprises ? Doit-on plutôt continuer à l'analyser uniquement comme une règle d'organisation de l'État? Au-delà de l'analyse de la façon dont le juge organisait jusque-là cette confrontation, l'objet de cette contribution est de s'interroger également sur le fondement et la portée du nouveau principe de neutralité de l'entreprise, issu de la loi du 8 août 2016.

\section{ABSTRACT}

Religious issues are more and more topical in a context paradoxically made of decreasing church attendance and reaffirmation of religious identities. In this context the question arises as to how reconcile the professional requirements and religious freedom in private organizations. Is it possible for private companies to resort to the French principle of laïcité to handle with the contemporary challenges they have to tackle? Or should this principle remain a rule applicable to the State organization only? Beyond an analysis of how courts have ruled this issue so far, this paper aims to deal with the basis and scope of the principle of neutrality in companies newly introduced by the Act of 8 August 2016. 
Comment gérer la diversité culturelle et religieuse dans des États marqués, tout à la fois, par une grande hétérogénéité des sensibilités religieuses liée à la mobilité - passée et contemporaine - des organisations et des personnes et par un double mouvement de montée du religieux et de sécularisation, dans un contexte imprégné, de surcroît, d'individualisme?

Quel mode de gestion privilégier, pour les pouvoirs publics comme pour les entreprises privées, dans l'espace public ${ }^{1}$ et dans les lieux privés : un aplanissement des différences, via la soumission de tous à une règle commune ou bien, au contraire, la reconnaissance de celles-ci, sur le modèle anglo-saxon, et, dans ce cas, jusqu'à quel point ${ }^{2}$ ?

S'il est bien une problématique centrale dans de nombreux secteurs d'activité et lieux de vie, c'est bien celle-là, comme en témoignent de multiples points de tension, même si l'on ne saurait les généraliser exagérément ${ }^{3}$ : port d'une croix dans les hôpitaux et compagnies aériennes (anglais) ${ }^{4}$; présence de crucifix dans les salles de classe (italiennes) ${ }^{5}$; port du burkini sur les plages (françaises) ${ }^{6}$; cours de natation obligatoires dans les écoles (suisses) ${ }^{7} \ldots$ Tel est notamment le cas de la France où se conjuguent plusieurs facteurs complexifiant cette problématique singulièrement en milieu de travail, ce que confirme un sondage récent ${ }^{8}$ et ce qui soulève des questions délicates pour lesquelles les managers de proximité se disent soucieux de réponses juridiques précises ${ }^{9}$. Ainsi, quelle attitude adopter face à une femme qui se présente voilée dans une entreprise ou qui le devient? Que répondre à un salarié qui sollicite des lieux et des temps de prière?

1. Loi $\mathrm{n}^{\circ}$ 2010-1192 du 11 octobre 2010, interdisant la dissimulation du visage dans l'espace public; CEDH, Gde ch., $1^{\text {er }}$ juill. 2014, no 43835/11, S.A.S. c/ France.

2. Bosset P., Réflexions sur la portée et les limites de l'obligation d'accommodement raisonnable en matière religieuse, Montréal, Commission des droits de la personne et des droits de la jeunesse, févr. 2005 : www.cdpdj.qc.ca/publications/religion_accommodement_avis.pdf [consulté le 26 juin 2017]

3. Pour un panorama, V. «Convictions religieuses sur le lieu de travail : variations nationales sur un principe de protection» [dossier], Rev. de droit comparé du travail et de la sécurité sociale, 2016/2.

4. CEDH, 15 janv. 2013, $\mathrm{n}^{\text {os }} 48420 / 10,59842 / 10,51671 / 10$ et 36516/10, Eweida et autres c/ Royaume-Uni.

5. CEDH, 12 mars 2011, n 30814/06, Lautsi c/ Italie.

6. CE, ord. réf., 26 août $2016, \mathrm{n}^{\text {os }} 402742,402777$.

7. CEDH, 10 janv. 2017, n² 29086/12, Osmanoğlu et Kocabaş c/ Suisse.

8. Étude Institut Randstad/ OFRE sur le fait religieux en entreprise, sept. 2016.

9. Huglo J.-G., «La Cour de cassation et le fait religieux dans l'entreprise : présentation des enjeux juridiques », Dr. soc. 2015, p. 682. 
Faut-il accepter des demandes d'aménagements d'horaires pour les fêtes religieuses ${ }^{10}$ ?

C'est ainsi que le premier facteur explicatif de ces difficultés françaises à concilier exigences professionnelles et liberté religieuse tient à la complexité du cadre normatif dans lequel cette conciliation doit être faite : un environnement source d'incertitudes juridiques, au-delà de l'évident respect des principes fondateurs et protecteurs de la liberté religieuse; des incertitudes générées par l'articulation complexe de ces deux règles que sont, d'une part, les normes garantissant l'exercice des droits et libertés fondamentales au travail ${ }^{11}$ et d'autre part, le principe de non-discrimination visé par les articles L. 1132-1 et L. 1133-1 du Code du travail. Ainsi l'affaire Baby Loup ${ }^{12}$ a-t-elle constitué le détonateur de la question de savoir comment combiner ces deux types de dispositions : application cumulative ou exclusive l'une de l'autre, l'enjeu étant lié au régime des discriminations plus rigoureux que celui applicable à l'exercice des libertés des salariés. D'ailleurs, telle est la raison pour laquelle, saisie d'une nouvelle affaire « du voile », la chambre sociale de la Cour de cassation ${ }^{13}$ a choisi, ainsi qu'on le verra, " de dépassionner le débat mais aussi de [le] dénationaliser », en saisissant la CJUE d'une question préjudicielle sur laquelle celle-ci vient de se prononcer ${ }^{14}$.

Pour autant, la délicate articulation des règles à appliquer ne constitue pas la seule cause des difficultés suscitées par la gestion religieuse en milieu de travail : y participe également cet autre paramètre complexe qu'est le principe de laïcité, dont la conventionnalité a été reconnue ${ }^{15}$, mais dont la teneur et, corrélativement, le périmètre n'en finissent pas d'être discutés ${ }^{16}$. Ainsi, comment définir ce principe? Quels en sont les fondements et les frontières ${ }^{17}$ ? Le terme a-t-il encore un sens, à l'heure où s'entrechoquent « laïcité-neutralité », « laïcité-positive », « laïcité-combat », certains parlant

10. CRÉPIN A., «Les entreprises privées face au fait religieux : entre fantasme et pragmatisme », Dr. soc. 2015, p. 694.

11. Art. L. 1121-1 du Code du travail.

12. Hennette Vauchez S. et Valentin V., L'affaire Baby Loup ou la nouvelle laïcité, Paris, LGDJ, 2014.

13. Cass. soc., 9 avr. 2015, $\mathrm{n}^{\circ} 13-19.855$.

14. CJUE, Gde ch., 14 mars 2017, aff. C-188/15, Asma Bougnaoui et ADDH c/ Micropole SA.

15. CEDH, 26 nov. 2015, n 64846/11, Ebrahimian c/ France.

16. La Morena F. DE, « Du principe de laïcité républicaine à l'application des valeurs de la laïcité dans l'entreprise », Dr. soc. 2015, p. 699.

17. Rivero J., « La notion juridique de laïcité », D. 1949, chron., p. 137. 
même de «laïcité en miettes ${ }^{18}$ », en raison de dispositifs pouvant être analysés comme « la fin de la laïcité fiscale ${ }^{19}$ »?

Dès lors, quel panorama du droit applicable peut-on faire, lequel est significatif de choix politiques, qui sont cependant plus ou moins clairement assumés? Plus précisément, les orientations récemment privilégiées par le législateur sont-elles révélatrices d'une volonté politique de modifier, sans le dire, les frontières du principe de laïcité dans ce champ particulier mais emblématique qu'est le travail ? Certes, c'est, en réalité, depuis 2004 que le législateur a élargi le périmètre du principe de laïcité, en y incluant, non pas seulement les agents publics, mais certains usagers ${ }^{20}$. Toutefois, un pas supplémentaire semble, selon nous, avoir été franchi en 2016, encore que le législateur ait pris soin de ne pas procéder frontalement à de tels changements. En témoigne la façon dont la loi du 8 août $2016^{21}$ a choisi de répondre aux demandes contemporaines de mise en retrait des convictions religieuses des salariés, pour mieux lutter contre les risques de communautarisme ${ }^{22}$ : une intervention qui est en effet axée sur la reconnaissance d'un nouveau principe de neutralité de l'entreprise, sans que soit évoqué le terme tabou de "laïcité » (1). Pour autant, la mise en ouvre de ce nouveau principe - dans lequel on est tenté de voir un avatar de celui de laïcité - ne semble pas aller de soi : bien au contraire, le choix d'une entreprise «neutre " s'avère lourd de sens (2).

\section{LA NEUTRALITÉ EN CONTEXTE DE TRAVAIL : REFLET DU PRINCIPE DE LAÏCITÉ}

Alors que le principe de laïcité obéit à un régime juridique précis, essentiellement issu de la loi du 9 décembre 1905 (art. 1 et 2), quel en est le périmètre?

18. Cabrillac R., Frison-Roche M.-A., Revet Th., Libertés et droits fondamentaux, Paris, Dalloz, 2012, p. 443.

19. Ibid.

20. Loi n 2004-228 du 15 mars 2004 encadrant, en application du principe de laïcité, le port de signes ou de tenues manifestant une appartenance religieuse dans les écoles, collèges et lycées publics. - Loi n ${ }^{\circ}$ 2010-1192 du 11 octobre 2010 interdisant la dissimulation du visage dans l'espace public. - V. Rapport de la commission « Laïcité et fonction publique » présidée par É. Zuccarelli, déc. 2016, p. 13.

21. Loi $n^{\circ}$ 2016-1088 du 8 août 2016 relative au travail, à la modernisation du dialogue social et à la sécurisation des parcours professionnels, art. 2.

22. MagGi-Germain N., «Communauté, communautarisme, religion et fait religieux : de la nécessité d'opérer quelques clarifications sémantiques et juridiques », Dr. soc. 2015, p. 674. 
A priori incongrue tant on pouvait considérer comme acquise l'idée selon laquelle - constituant un principe fondateur de la République française - ce principe ne s'imposait qu'en cas de poursuite d'une mission de service public, cette interrogation est devenue majeure. En cause : un contexte difficile, favorable à des demandes d'élargissement du principe hors de son champ originel, soit les seuls agents du service public sauf exception ${ }^{23}$. En jeu : la préservation de la liberté d'expression des convictions religieuses dans les organismes de droit privé ne poursuivant pas une telle mission, tant il apparaît que, par un système de vases communicants, là où ne s'applique pas le principe de laïcité doit prévaloir la liberté religieuse et inversement.

Entre laïcité et liberté d'exprimer ses convictions religieuses, ce sont alors de véritables querelles de frontières qui sont apparues en droit du travail, dans un contexte propice à une instrumentalisation de ce qui devrait pourtant demeurer un principe reposant sur ces trois piliers : liberté de conscience, pluralisme religieux et neutralité de l'État. Et, au final, c'est par une espèce de tour de "passe-passe » qu'il a été choisi de résoudre ce différend, sans que l'on doive minimiser l'impact de ce choix. En effet, si, hier, le juge avait choisi de limiter le champ du principe de laïcité pour préserver celui de la liberté religieuse (1.1), c'est, aujourd'hui, le législateur qui a choisi de limiter le champ de la liberté religieuse au nom d'une nouvelle neutralité de l'entreprise, sachant que "derrière "neutralité", il convient (très certainement) de lire "laïcité" ${ }^{4} »(1.2)$.

\subsection{HIER : UN CANTONNEMENT DE LA LAÏCITÉ AU NOM DE LA LIBERTÉ RELIGIEUSE}

Quel est le champ d'application du principe de laïcité ? Alors qu'elle ne semblait guère discutable pour les tenants d'une approche classique, la réponse à cette question a fluctué au cours de la «saga »Baby Loup ${ }^{25}$ au point de rendre opaque la signification même du principe : règle d'organisation de l'État à valeur constitutionnelle et/ou simple option idéologique offerte aux personnes privées? Génératrice d'intenses polémiques juridiques et politiques, la question fut enfin tranchée - sur le plan judiciaire au moins après pas moins de cinq décisions destinées à clarifier les champs respectifs des principes de laïcité et de liberté religieuse.

23. V. supra, note 20.

24. En ce sens, Mouly J., «Le principe de neutralité dans l'entreprise », D. 2016, p. 1992.

25. Champeaux F., « Il fallait sauver le soldat Baby Loup », SSL, n 1637, p. 12. 
Rappelons - même si elle est encore dans tous les esprits - que l'affaire débutât le 19 décembre 2008, lorsque Fatima A., salariée d'une crèche associative, fût licenciée pour faute grave, suite au refus d'ôter son voile. Alors que la directrice justifiait cette décision par l'objectif de "neutralité » poursuivi par la crèche, la salariée dénonça un licenciement abusif, réclamant en outre plus de 100000 euros de dommages et intérêts.

Donnant raison à la direction, les juges prud'homaux ${ }^{26}$ puis ceux de la cour de Versailles ${ }^{27}$ jugèrent le licenciement justifié sur la base d'argumentations cependant différentes. En effet, alors que les premiers évoquèrent la violation des principes de laïcité et de neutralité visés par le règlement intérieur et applicables au personnel de l'association eu égard à son activité de « service public », c'est une logique plus classique qu'adoptèrent les seconds : s'appuyant sur les articles L. 1221-1 et L. 1321-3 du Code du travail, ils conclurent que les restrictions apportées à la liberté religieuse étaient justifiées par la nature de la tâche à accomplir et proportionnées au but recherché.

Coup de théâtre le 19 mars 2013 : désavouant la cour de Versailles, c'est en faveur de la salariée que la Chambre sociale trancha le litige en décidant que, « s'agissant d'une crèche privée, le licenciement constituait une discrimination en raison des convictions religieuses de la salariée et devait être déclaré nul ${ }^{28}$ ». Ainsi, non seulement le fait religieux n'est pas exclu dans une organisation privée, mais toute atteinte injustifiée à cette liberté est discriminatoire. Quant au principe de laïcité, il n'a pas sa place dans la sphère privée, exception faite des agents des personnes privées chargées d'une mission de service public ${ }^{29}$.

Étonnamment médiatisée avant même qu'elle ne soit rendue, c'est une analyse inverse que la cour de renvoi de Paris ${ }^{30}$ retint puisqu'elle décida de confirmer le licenciement litigieux : l'association pouvait être qualifiée « d'entreprise de conviction en mesure d'exiger la neutralité de ses employés » et la façon dont cette obligation était formulée dans le règlement intérieur

26. Cons. prud'h. Mantes-la-Jolie, 13 déc. 2010 , n 10/587, D. 2011, p. 85 ; Dr. soc. 2011, p. 779, note Boualili H.; RDT 2011, p. 182, obs. ADAm P.

27. CA Versailles, 27 oct. 2011, no 10/5642, D. 2012, p. 901, obs. LokieC P. et PorTA J. ; Dr. soc. 2011, p. 1186, note GaUdu F.

28. Cass. soc., 19 mars 2013, no 11-28.845, D. 2013, p. 956, avis AldiGÉ B. ; D. 2013, p. 963 , note Mouly J. ; D. 2013, p. 1026, obs. Lokiec P. et PorTa J. ; Dr. soc. 2013, p. 388, étude Dockès E. ; Dr. soc. 2014, p. 100, étude Laronze F. ; RDT 2013, p. 385, étude Adam P.; RDT 2014, p. 94, étude CALVÈs G.; SSL, n 1577, p. 8, étude SCHWARTZ R.

29. Cass. soc., 19 mars 2013, $\mathrm{n}^{\circ} 12-11.960$.

30. CA Paris, 27 nov. 2013, no 13/02981, D. 2014, p. 65, étude Mouly J. ; Dr soc. 2014, p. 4, étude Ray J-E. ; Cah. soc., $1^{\text {er }}$ févr. 2014, n 260, p. 90, étude AKANDJi-Kombé J.-F. ; RDT 2014, p. 391, étude Antonmattei P. H. 
était «précise [...] et n'avait pas la portée d'une interdiction générale », de telle sorte que les restrictions apportées à la liberté religieuse respectaient les exigences de nécessité et de proportionnalité et n'étaient pas discriminatoires au sens de l'article L. 1132-1 du Code du travail.

Suivant sur ce point la Chambre sociale, c'est un retour aux sources que l'Assemblée plénière ${ }^{31}$ décida d'opérer en confirmant, d'une part, le refus d'un élargissement direct du principe de laïcité en l'absence de mission de service public, dans le droit fil de l'analyse prônée par la Commission nationale consultative des droits de l'homme ${ }^{32}$ et par le Conseil d'État ${ }^{33}$, et en récusant, d'autre part, le recours à l'entreprise de « conviction » : une solution qui, en cas de poursuite d'une mission d'intérêt général, aurait permis une extension indirecte du principe de neutralité religieuse et dont le rejet a ainsi permis d'écarter tout risque de voir coexister « deux laïcités » : « la première, constitutionnelle, publique, obligatoire et propre à l'État et à ses services publics, la seconde, contractuelle, privée et propre à certaines personnes morales de droit privé qui [auraient] choisi de s'y référer ${ }^{34} »$. Pour autant, c'est à la validité du licenciement qu'a cependant conclu l'Assemblée plénière : en effet, elle a approuvé la cour d'appel de Paris qui - ayant apprécié les conditions de fonctionnement d'une association de dimension réduite, employant seulement dix-huit salariés, qui étaient ou pouvaient être en relation directe avec les enfants et leurs parents - avait pu en déduire que la restriction à la liberté de religion édictée par le règlement répondait aux canons de justification et de proportionnalité imposés par la loi. Conséquence? C'est à une réaffirmation du principe de la liberté religieuse dans les organisations privées ne poursuivant pas une mission de service public qu'aboutit cette remise en ordre du principe de laïcité et, ce faisant, à un cantonnement de celui-ci au nom de celle-là : un raisonnement dont, cependant, la logique vient d'être déstabilisée par la loi du 8 août 2016 puisque, désormais, c'est au contraire de cantonnement de la liberté religieuse au nom d'un nouveau principe de neutralité de l'entreprise qu'il faut parler si celle-ci en fait le choix; un nouveau principe puisant sa source dans l'article L. 1321-2-1 du Code du travail, dans lequel on peut voir un reflet du principe de laïcité.

31. Cass. ass. plén., 25 juin 2014, n 13-28.369, SSL, n 1637, p. 12, étude Champeaux F.; AJDA 2014, p. 1293, étude PASTOR J.-M.

32. CNCDH, Avis sur la laïcité, 26 sept. 2013.

33. France. Conseil d'État, Étude demandée par le Défenseur des droits le 20 septembre 2013, adoptée par l'Assemblée générale du Conseil d'État le 19 décembre 2013.

34. Dieu F., « L'affaire Baby-Loup : quelles conséquences sur le principe de laiicité et l'obligation de neutralité religieuse? », JCP A 2014, 2114. 


\subsection{AUJOURD'HUI : UN CANTONNEMENT DE LA LIBERTÉ RELIGIEUSE AU NOM DE LA NEUTRALITÉ DE L'ENTREPRISE}

Après plusieurs tentatives destinées à un élargissement du devoir de neutralité notamment aux crèches privées ${ }^{35}$, c'est par un autre biais que la loi du 8 août 2016 a réalisé l'objectif d'extension du périmètre de l'interdiction des signes religieux. En effet, s'éloignant de la proposition originellement faite par le rapport Badinter ${ }^{36}$ et qui avait été particulièrement controversée, le législateur a opté pour un élargissement du champ du règlement intérieur en décidant qu'il "peut contenir des dispositions inscrivant le principe de neutralité et restreignant la manifestation des convictions des salariés si ces restrictions sont justifiées par l'exercice d'autres libertés et droits fondamentaux ou par les nécessités du bon fonctionnement de l'entreprise et si elles sont proportionnées au but recherché » (C. trav., L. 1321-2-1).

Dès lors, de deux choses l'une.

La première hypothèse sera celle dans laquelle une entreprise ne s'est pas dotée d'une telle norme interne, auquel cas prévaudra le principe de la liberté religieuse, sauf à pouvoir y apporter des restrictions justifiées par la nature de la tâche à accomplir et proportionnées au but recherché, une telle conciliation entre exigences religieuses et professionnelles devant se faire en fonction des pratiques en cause : port de signes religieux ${ }^{37}$, absences pour raisons religieuses ${ }^{38}$, demandes d'aménagement des conditions d'exécution du travail pour raisons religieuses ${ }^{39}$, non-respect d'obligations contractuelles pour motif religieux ${ }^{40}$, actes de prosélytisme ${ }^{41}$.

35. Proposition de loi visant à étendre l'obligation de neutralité à certaines personnes ou structures privées accueillant des mineurs et à assurer le respect du principe de laïcité, adoptée en première lecture avec modifications par l'Assemblée nationale le 13 mai 2015. Déposée au Sénat le 25 octobre 2011 par Mme Françoise Laborde et plusieurs de ses collègues, cette proposition de loi avait été adoptée en première lecture par le Sénat le 17 janvier 2012.

36. Dumortier G., PéCAut-Rivolier L., « Naissance des principes essentiels du droit du travail », RDT 2016, p. 79.

37. Cass. soc., 9 avr. 2015, $\mathrm{n}^{\circ} 13-19.855$.

38. Cass. soc., 16 déc. 1981, Bull. civ. V, n 968 ; CA Paris, 10 janv. 1989, n 35228/87, $n^{\circ} 35180 / 87$ Vs CA Paris, 25 janv. 1995, n³ 31766/94.

39. CE, 16 févr. 2015, n 375724.

40. Cass. soc, 24 mars 1998, Bull. civ. V, n 171. Comp. Cass soc., 12 juill. 2010, n 08-45.509. Rappr., en cas d'inexécution d'une obligation légale, Cass. soc, 29 mai 1986, n 83-45.409.

41. CA Toulouse, 21 mai 2008, n 07/01305; CA Aix-en-Provence, ch. 9, 15 févr. 1989, $n^{\circ} 875141$. 
Le second cas sera celui d'une entreprise ayant décidé de se présenter comme «neutre»: une option désormais ouverte par l'article précité, dont l'adoption s'est ainsi faite dans un contexte marqué, non seulement par l'affaire Baby Loup, mais également par la polémique générée par les 61 principes « essentiels » du droit du travail identifiés par la commission Badinter, auxquels il fût globalement reproché d'être insécurisants sur le plan juridique ${ }^{42}$ et, s'agissant plus spécifiquement du $6^{\mathrm{e}}$ principe, d'ouvrir la voie au communautarisme dans l'entreprise ${ }^{43}$.

Cela étant, tant s'en faut qu'une telle option pour un environnement de travail «neutre » aille de soi, alors même que cette disposition, introduite par le Sénat, fut largement adoptée sans pour autant faire consensus ${ }^{44}$. Bien au contraire, se pose la question de savoir si l'on se trouve en présence d'un simple effet d'annonce ou d'une disposition périlleuse pour les libertés.

C'est ainsi qu'il est loisible d'estimer qu'il ne s'agit là que d'une mesure d'affichage en raison du caractère limité des restrictions tolérées, les entreprises ne disposant finalement que de faibles marges de manœuvre. En effet, - devant éviter les risques d'une potentielle discrimination, de l'atteinte à la liberté d'expression des salariés et à leur liberté religieuse - elles devraient être confrontées, non seulement à la justification des restrictions permises, mais également à leur proportionnalité par rapport au but recherché : une double exigence d'autant plus lourde de conséquences qu'aujourd'hui imprécise dans ses contours.

Ainsi, du point de vue des justifications possibles et au-delà des traditionnelles exigences de sécurité et de préservation de la santé, quid - au titre de la formule nouvelle mais indéterminée des «autres droits et libertés fondamentaux »-de la liberté de conscience (des collègues et/ou des clients) et de la liberté d'entreprendre ? Quid également des « nécessités du bon fonctionnement de l'entreprise », nouvelle formule issue du rapport Badinter, dont la portée semble plus large que la traditionnelle référence à « la nature des tâches à effectuer »? Comment entendre de telles «nécessités» (au regard de l'image de l'entreprise, des exigences de la clientèle ou bien des relations entre salariés ?) et de quelle façon les mesurer ${ }^{45}$ ?

42. V. IZARD S., « Les 61 principes disparaissent du Code du travail », SSL, n ${ }^{\circ} 1715$, p. 3.

43. Moullot P., «Non, la loi travail n'introduit pas "le fait religieux" dans l'entreprise», Libération, 24 mars 2016.

44. Demande de retrait du principe, dans un communiqué commun du 19 juillet 2016 de l'Observatoire de la laïcité et de la Commission nationale consultative des droits de l'homme.

45. Sur ces questionnements, V. Pagnerre Y., " Principe de neutralité dans l'entreprise », Dr. soc. 2016, p. 880. 
Par ailleurs - et au regard cette fois du nécessaire respect de proportionnalité à la lumière du but recherché - comment mener cette analyse ? Ne devrait-elle pas être menée, «dans sa plus pure expression»: "rationae personae quant aux salariés concernés, rationae materiae quant aux fonctions et tâches en cause, rationae loci quant aux lieux de travail dans et hors de l'entreprise et rationae temporis quant aux différents temps de travail, de pause, de restauration ${ }^{46} »$.

Mais si, pour certains, il ne s'agit donc que d'une « innovation technique, [...] plus "incantatoire" qu'"opératoire" 47 ", d'autres évoquent une disposition qui serait, au contraire, attentatoire au principe de laïcité et dangereuse pour les libertés ${ }^{48}$. En effet, telle est la situation qui pourrait résulter de l'affirmation d'une "neutralité » caractérisée par un périmètre illimité à la fois au regard des secteurs concernés (pas de limitation à des lieux particuliers, tels ceux dédiés à l'accueil des mineurs) et du point de vue des convictions concernées (pas de limitation aux seules convictions religieuses des salariés, mais extension possible aux opinions politiques, syndicales...). Voilà pourquoi l'évolution induite par la loi du 8 août 2016 devrait être lourde de sens à deux égards. D'abord, au regard de sa légalité même, la conformité de l'article L. 1321-2-1 aux textes nationaux et internationaux, n'allant pas de soi ${ }^{49}$; ensuite, du point de vue de la reconnaissance ainsi opérée d'entreprises «neutres », dont la portée s'avère source d'interrogations. En cause : le fait que, dans un contexte d'hypertrophie du principe de neutralité, se pose fondamentalement - pour les pouvoirs publics comme pour les entreprises privées - la question de savoir comment concilier exigences professionnelles et liberté religieuse, dans une société plurielle où la gestion de la diversité constitue un pari social et un enjeu d'entreprise. Au-delà, on peut encore s'interroger sur l'impact que risque d'avoir la reconnaissance de ce principe de neutralité qui - s'il devait s'analyser comme un avatar du principe de laïcité - pourrait en modifier le sens. En effet, jusque-là classiquement entendu comme la «neutralité de l'État et la garantie de liberté offerte à toutes les croyances », ce principe pourrait être désormais compris dans le sens d'une prohibition : celle opposée à la liberté religieuse. Plus précisément, c'est, selon certains, de «monstre juridique » qu'il s'agirait ou,

46. Pagnerre Y., précit.

47. Adam P., Le Friant M., Pécaut-Rivolier L., Tarasewicz Y., « La religion dans l'entreprise. L'art (difficile) des limites », RDT 2016, p. 532.

48. Communiqué commun du 19 juillet 2016 de l'Observatoire de la laïcité et de la Commission nationale consultative des droits de l'homme.

49. V. infra. 
à tout le moins, d'une " cacophonie complète », en raison d'une "neutralité religieuse dont la définition [risque de changer] selon les entreprises ${ }^{50} »$.

\section{L'ENTREPRISE « NEUTRE ॥? UNE OPTION DOUBLEMENT PROBLÉMATIQUE}

Pour deux raisons, l'admission d'une entreprise souhaitant se rendre «neutre " peut se révéler incertaine. La première est de nature juridique, les fondements du nouvel article L. 1321-2-1 du Code du travail pouvant paraître fragiles, non seulement au regard de la jurisprudence européenne, mais également de la Constitution française (2.1). La seconde est d'ordre entrepreneurial, les entreprises se disant "éthiques » pouvant se retrouver en pleine contradiction entre l'aspiration à la neutralité et une gestion des diversités requise par une démarche dite « socialement responsable » (2.2). Certes, le rapprochement ainsi opéré entre l'instauration légale d'un principe de neutralité dans l'entreprise et le possible engagement de celle-ci dans ce type de démarche gestionnaire peut étonner. Il n'en est pas moins justifié dans la mesure où, sans parler des origines religieuses du concept de RSE ${ }^{51}$, la mise en œuvre de celui-ci peut englober la thématique du fait religieux dans l'entreprise, via celle de la gestion des diversités : la célèbre charte du groupe Paprec l'illustre qui s'intitule, sans crainte du paradoxe, « Charte de la laïcité et de la diversité », au nom d'un mieux «vivre ensemble».

\subsection{DES FONDEMENTS JURIDIQUES FRAGILES}

La possibilité désormais donnée aux entreprises de se déclarer «neutres » - pour ne pas employer la terminologie polémique d'entreprise de «tendance laïque ${ }^{52} »-$ pourrait-elle se révéler contraire au droit communautaire ? Tel est

50. Gille J.-P., in Rapport $n^{\circ} 3909$ sur le projet de loi de modernisation du droit du travail par Christophe Sirugue, Paris, Assemblée nationale, 30 juin 2016, p. 40.

51. Acquier A., Gond J-P, Igalens J., « Des fondements religieux de la responsabilité sociale de l'entreprise à la responsabilité sociale de l'entreprise comme religion », Cahier de recherche, $\mathrm{n}^{\circ}$ 166, mai 2005 (Centre de recherche en gestion, Toulouse).

52. Gaudu F., «L'entreprise de tendance laïque », Dr. soc. 2011, p. 1186. Pour un panorama des opinions divergentes quant «à la question de savoir si une entreprise privée peut se prévaloir d'une éthique fondée sur une conviction "laïque" ou de simple neutralité pour imposer à ses employés une interdiction de porter tout signe ostentatoire de religion », V. le rapport de L. Truchot, 16 juin 2014, p. 48 et s. (Cass. ass. plén., 25 juin 2014, $\mathrm{n}^{\circ}$ 13-28.369, L. c/ Assoc. Baby-Loup). 
le sentiment qui est né de la confrontation de deux affaires, l'une belge, l'autre française, dont la CJUE a été saisie : deux affaires relatives à des salariées en contact avec la clientèle et licenciées pour refus d'ôter leur voile ${ }^{53}$; deux affaires qui ont, chacune, donné lieu à une question préjudicielle relative à la portée de l'article 4 \& 1 de la directive 2000/78/CE du 27 novembre 2000, mais qui ont conduit à des conclusions différentes des avocates générales ${ }^{54}$.

D’un côté, dans l'affaire belge ${ }^{55}$, l'avocate générale Kokott avait conclu à l'absence de discrimination directe fondée sur la religion (dès lors que le règlement litigieux interdisait «aussi bien les signes politiques et philosophiques que les signes religieux visibles, sans viser une ou plusieurs religions déterminées »), et estimé que, dans le cas où une discrimination indirecte serait retenue, elle pouvait être justifiée par la «politique légitime de neutralité », liée à la diversité des clients, souhaitée par l'entreprise.

À l'inverse, dans l'affaire française ${ }^{56}$, l'avocate générale Sharpston estimait que la salariée avait été victime d'une discrimination directe de surcroît injustifiable, du fait notamment de l'inexistence d'une « exigence professionnelle essentielle et déterminante » (EPED), le souhait de la clientèle de ne plus voir des prestations assurées par une salariée voilée ne pouvant être ainsi qualifié.

Ce faisant, il est certain que la reprise, par la CJUE, de l'argumentation développée par l'avocate Sharpston aurait fragilisé l'article L. 1321-2-1 du Code du travail. La raison en est que, pour celle-ci, c'est « sous l'angle de la discrimination et non, comme le fait la $\mathrm{CEDH}$, sous [celui] de la restriction à un droit ou à une liberté ${ }^{57}$ » que la question du voile au travail doit être envisagée, ce dont il ressort que les dérogations possibles ne sont pas identiques ${ }^{58}$. Dès lors, une telle démarche axée sur le droit des discriminations aurait eu pour effet de conclure qu'un « règlement de travail

53. Dans l'affaire belge, une réceptionniste avait été licenciée pour avoir violé l'obligation de neutralité inscrite dans le règlement de travail de l'entreprise en portant un foulard pour raisons religieuses. Dans le cas français, une salariée est licenciée par son employeur, afin de tenir compte du souhait d'un client de ne pas être confronté à une salariée voilée.

54. Moizard N., «Les toges européennes se déchirent sur le voile au travail », RDT 2016, p. 569.

55. Aff. C-157/15, Samira Achbita, conclusions de l'avocat général J. Kokott présentées le 31 mai 2016.

56. Aff. C-188/15, Asma Bougnaoui, conclusions de l'avocat général E. Sharpston présentées le 13 juillet 2016.

57. Laulom S., « Pour une interprétation plus fidèle de la directive 2000/78. Les conclusions de l'avocate générale dans l'affaire française sur le port du voile au travail », SSL, $\mathrm{n}^{\circ} 1733$, p. 10.

58. Concl. Sharpston, \& 63. 
[...] qui interdit aux travailleurs de porter des signes ou tenues vestimentaires religieux lorsqu'ils sont en contact avec la clientèle de leur entreprise constitue une discrimination directe $[\ldots]^{59}$ », et que, si cette discrimination était qualifiée d'indirecte, il n'est pas certain qu'une telle mesure passe le test de proportionnalité, même si elle franchissait celui de sa légitimité. Conséquence? Une telle analyse n'aurait pu que s'appliquer à un règlement intérieur qui, tel celui fondé sur l'article L. 1321-2-1 du Code du travail, imposerait aux salariés d'une entreprise une obligation totale de neutralité.

Or, il s'avère que la solution finalement retenue par la CJUE le 14 mars 2017 devrait être de nature à atténuer ces risques d'incompatibilité de l'article L. 1321-2-1 du Code du travail avec le droit communautaire ${ }^{60}$. La raison en est que le juge a admis que :

«L'interdiction de porter un foulard islamique, qui découle d'une règle interne d'une entreprise privée interdisant le port visible de tout signe politique, philosophique ou religieux sur le lieu de travail, ne constitue pas une discrimination directe $[. .$.$] . En revanche, une telle interdiction$ est susceptible de constituer une discrimination indirecte s'il est établi que l'obligation en apparence neutre qu'elle prévoit entraîne [...] un désavantage particulier pour les personnes adhérant à une religion ou à des convictions données, [...] une telle discrimination indirecte [pouvant être cependant] objectivement justifiée par un objectif légitime, tel que la poursuite par l'employeur, dans ses relations avec ses clients, d'une politique de neutralité [...], pourvu que les moyens de réaliser cet objectif soient appropriés et nécessaires ${ }^{61} »$.

Quant au cas dans lequel aucune règle interne de ce type n'aurait été adoptée, il est jugé que la volonté d'un employeur de tenir compte des souhaits du client de ne plus voir ses services assurés par une salariée portant un voile ne saurait être considérée comme une exigence professionnelle de nature à écarter l'existence d'une discrimination. Conséquence ? Sous le contrôle du juge national, un règlement intérieur peut donc limiter le port de signes religieux, au nom d'une politique de neutralité vis-à-vis de la clientèle, sous réserve que cette restriction soit limitée aux salariés en relation avec celle-ci.

Si les risques de contrariété avec le droit communautaire semblent ainsi circonscrits, et que l'on peut même estimer que «cette décision pourrait

59. Concl. Sharpston, \& 108.

60. CJUE, Gde ch., 14 mars 2017, aff. C-157/15 et C-188/15 ; LAUlom S., « Un affaiblissement de la protection européenne contre les discriminations », SSL, n 1762, p. 6.

61. CJUE, communiqué de presse, n 30/2017, 14 mars 2017. 
donner une assise supplémentaire au nouvel article L. 1321-2-1 du Code du travail $^{62}$ », le principe de neutralité «à la française », n'est-il pas cependant fragile à deux autres égards? Telle est l'opinion de certains qui soulignent que, "même si le texte a franchi le cap du Conseil constitutionnel, il a tout à redouter [non seulement] d'une éventuelle QPC, [mais aussi] de la Cour de Strasbourg ", qui « reconnaît [...] aux salariés le droit de manifester leurs convictions religieuses dans l'entreprise ${ }^{63} »$. Le fait est, plus précisément, qu'en raison de l'absence de définition juridique de la "neutralité ", on ne saurait a priori exclure une saisine du Conseil constitutionnel par voie de question prioritaire de constitutionnalité, d'autant que, dans sa décision n 2016-736 DC du 4 août 2016, celui-ci ne s'est prononcé que sur quelques articles, renvoyant explicitement son appréciation sur les autres (notamment l'article 2) à d'éventuels recours. En jeu : le risque d'éventuelles discriminations liées au fait que, s'appliquant indifféremment à toutes les convictions des salariés, le principe de neutralité englobe celles de nature politique, syndicale ou mutualiste dont, pourtant, la légitimité est expressément protégée par la loi.

Ce ne sont pas là, cependant, les seules interrogations suscitées par la possibilité désormais offerte à une entreprise de se déclarer «neutre». En effet, c'est une autre question, bien différente, qui doit être également évoquée, même si elle ne relève pas de préoccupations essentiellement juridiques: elle est relative au risque d'incohérence d'une entreprise se voulant, tout à la fois, «neutre » et «éthique » au titre d'un comportement « socialement responsable».

\subsection{ENTRE NEUTRALITÉ DE L'ENTREPRISE ET GESTION DES DIVERSITÉS : QUELLE COHÉRENCE?}

Entre indifférence et management des particularismes : quelle option privilégier pour les entreprises? À l'évidence, il s'agit là d'un dilemme auquel nombre d'entre elles sont confrontées comme en témoignent, d'un côté, l'objectif de neutralité poursuivi par certaines mais, d'un autre, celui d'une gestion des pluralités - d'ailleurs parfois poursuivi par les mêmes - : un second but générateur de nouvelles pratiques professionnelles, d'autant plus fréquemment visé que désormais stimulé par différentes contraintes,

62. «Restrictions au port de signes religieux en entreprise : la CJUE pose ses conditions », Liaisons Sociales Quotidien, 16 mars 2017.

63. MOULY J., préc. 
notamment juridiques. Le fait est que c'est un contexte normatif mais également socioéconomique en pleine évolution qui explique l'émergence de nouvelles pratiques entrepreneuriales tendues vers une gestion des diversités au travail, mais qui sont susceptibles de télescoper l'objectif de neutralité évoqué par l'article L. 1321-2-1 du Code du travail.

C'est ainsi que les pressions exercées sur les entreprises pour les inciter à adopter ce type de pratiques sont, d'abord, d'ordre juridique, comme en témoigne ce double phénomène : un alourdissement des obligations légales en matière de lutte contre les discriminations, couplé à la montée en puissance de l'obligation de reporting extra financier, sans cesse renforcée en France depuis 2001 et qui est désormais relayée au niveau communautaire ${ }^{64}$.

En outre, les enjeux, pour les entreprises, sont également d'ordre économique et en rapport avec leur «image ». Enjeux économiques, en raison du lien pouvant être établi entre promotion de la diversité sur le lieu de travail et performance de l'organisation ${ }^{65}$. Mais également enjeu de réputation, dès lors qu'une démarche " diversité » peut valoriser « l'image » de l'entreprise la mettant en œuvre dans une logique de stratégie de communication ${ }^{66}$.

Voilà pourquoi, à l'heure de la responsabilité sociale des entreprises, certaines d'entre elles adoptent de nouveaux comportements révélateurs d'un objectif de gestion des diversités, celui-ci pouvant se traduire par des mesures de lutte contre les discriminations, de promotion de l'égalité des chances, voire de valorisation des différences, selon les trois mouvements suivants:

Premier mouvement révélateur d'une attention portée à une gestion des diversités au travail : l'impact de la labellisation sur les pratiques d'entreprise, ainsi qu'en témoignent l'adoption possible du label diversité $e^{67}$ ou une éventuelle adhésion à la charte de la diversité.

Deuxième type de pratique entrepreneuriale : la teneur parfois donnée par les partenaires sociaux à leurs accords collectifs, qu'ils soient conclus

64. Directive 2014/95/UE du 22 octobre 2014 modifiant la directive 2013/34/UE en ce qui concerne la publication d'informations non financières et d'informations relatives à la diversité par certaines grandes entreprises et certains groupes.

65. Montargot N., Peretti J-M, « Regards de responsables sur les notions d'égalité, nondiscrimination et diversité », Management \& Avenir, 2/2014, p. 183-200.

66. BÉBÉAR C., Rapport sur la non-discrimination et la gestion de la diversité dans les entreprises en France, déc 2004. - Bruna M. G, « La promotion de la diversité en entreprise. Un vecteur de performance globale », Cadres-Cfdt, $\mathrm{n}^{\circ}$ 459, 2014.

67. Décret $n^{\circ}$ 2008-1344 du 17 décembre 2008 relatif à la création d'un label en matière de promotion de la diversité et de prévention des discriminations dans le cadre de la gestion des ressources humaines et à la mise en place d'une commission de labellisation, art. 1. 
au niveau national ou international. C'est ainsi qu'au niveau national, «les conventions collectives [branche ou entreprise] peuvent également faciliter le respect des prescriptions ou des usages religieux en prévoyant des jours fériés ou des autorisations d'absence à l'occasion d'événements à connotation religieuse ${ }^{68} »$, encore que la question du fait religieux dans l'entreprise soit jugée « difficile » par certains syndicats ${ }^{69}$. En outre, des initiatives peuvent être également prises au niveau interprofessionnel, comme en témoigne l'adoption de l'accord national interprofessionnel (ANI) du 12 octobre 2006 relatif à la diversité dans l'entreprise ${ }^{70}$. Rappelons en effet que ce texte souligne qu'«au-delà des principes éthiques [constituant] le fondement de leur démarche, les entreprises ont un intérêt économique et social à mieux accueillir la diversité », ce qui suppose "de faire évoluer les mentalités [et] de lutter contre les stéréotypes », cette diversité devant être largement entendue puisque pouvant être "sociale, culturelle et ethnique » et recouper les « discriminations directes ou indirectes, conscientes ou inconscientes, relevant des origines réelles ou supposées ». Il faut noter cependant, que, «bien que l'origine ethnique, culturelle et sociale soit au cœur de cet ANI, elle n'est que peu traitée dans les accords étudiés portant sur la diversité [à la différence des] composants de la diversité déjà encadrés par la législation $[\ldots]$ : égalité entre femmes et hommes, emploi et conditions de travail des salariés âgés et des travailleurs handicapés ${ }^{71} »$.

Quant aux initiatives supranationales, il s'agit de celles traduites par la mise en œuvre d'accords-cadres internationaux conclus par des entreprises multinationales désireuses de sécuriser les conditions de leur production, y compris chez leurs partenaires. Si le contenu de ces accords, initialement focalisé sur la protection des droits fondamentaux au travail, a été élargi à de nouvelles thématiques (accompagnement social des restructurations...) ${ }^{72}$,

68. Morin C., "Le salarié et la religion: les solutions de droit du travail», JCP A 2005, p. 1145.

69. V. par ex. le point de vue de la CGT in ARnoult-Brill V. et SimOn G., Le fait religieux dans l'entreprise, nov. 2013 (Les avis du Conseil économique, social et environnemental), p. 30.

70. Verkindt P.-Y., « L'ANI sur la diversité, un accord à l'honneur de la démocratie sociale », SSL, $\mathrm{n}^{\circ} 1287$, p. 6.

71. Garner H. et Recoules M., Étude de 80 accords d'entreprise sur la diversité, Paris, DARES, juin 2014 (Document d'études DARES; 182).

72. Hallou Y., Analyse comparative et critique de 26 accords-cadres internationaux conclus par des entreprises transnationales, Paris, Min. des affaires étrangères/ORSE, 12 nov. 2010. - Daugareilh I., "Accord-cadre international», in Postel N., Sobel R. (dir.), Dictionnaire critique de la RSE, Villeneuve-d'Ascq, Presses universitaires du Septentrion, 2013, p. 17-20. 
il n'est pas certain cependant que la thématique de la gestion des diversités, notamment religieuse, occupe une place majeure.

Voilà pourquoi, en troisième et dernier lieu, ce sont les initiatives prises par les employeurs eux-mêmes qui semblent les plus révélatrices d'un objectif de gestion des diversités dans leurs organisations, les propositions ayant été faites par l'ANDRH jouant ici un rôle clef parce qu'évoquant explicitement la thématique de la religion au travail ${ }^{73}$. Rappelons en effet qu'au motif que «le risque des entreprises est celui de l'uniformité de pensée et d'attitude » et que "la diversité doit s'analyser comme l'une des conditions de leur compétitivité », cette association avait proposé de mettre en débat un certain nombre de propositions telles que la mise en place d'indicateurs " permettant de mesurer les minorités visibles et non visibles ou l'ouverture d'un débat national sur le positionnement des jours fériés » afin de mieux concilier « la neutralité nécessaire », la «liberté de conscience » et l'«accroissement de la compétitivité »: des propositions qui, en réalité, n'étaient pas novatrices puisque déjà préconisées il y a plusieurs années par la Commission Stasi ; des recommandations réitérées en 2010 par la Mission d'information sur la pratique du port du voile intégral; mais des préconisations non suivies d'effets puisque, bien au contraire, c'est la possibilité d'opter pour un environnement neutre que la loi du 8 août 2016 a offerte aux entreprises le souhaitant.

Est-ce à dire qu'entre aspirations en faveur d'une telle neutralité de l'environnement de travail et requêtes des différentes parties prenantes d'une entreprise en faveur d'une gestion «inclusive » des diversités, c'est la première option qui l'a définitivement emporté ? Rien n'est moins sûr à l'heure où les exigences de responsabilité sociétale imposées aux entreprises n'ont jamais été aussi importantes, plaçant celles-ci au milieu du gué ${ }^{74}$.

73. Association nationale des DRH, Propositions 2012 de l'ANDRH. Le DRH et les enjeux de la compétitivité, 29 juin 2012.

74. Pour un panorama des actions promues par les pouvoirs publics en France et au niveau international pour encourager la responsabilité sociale et environnementale des entreprises, V. Rapport du Gouvernement relatif à l'application par les entreprises des dispositions de l'article L. 225-102-1 du code de commerce et du décret " Grenelle II », sept. 2016 : http:// www.tresor.economie.gouv.fr/File/432219 [consulté le 26 juin 2017] 